\title{
Efeitos colaterais do metilfenidato
}

\section{Side Effects of Methylphenidate}

\author{
GiusePpe PASTura ${ }^{1}$ \\ Paulo Mattos ${ }^{2}$
}

\section{Resumo}

Objetivos: Revisar os principais efeitos colaterais do metilfenidato, em curto e longo prazos, no tratamento de crianças com Transtorno do Déficit de Atenção (TDAH). Fo n te s de dados: Revisão de literatura nos últimos doze anos através de pesquisa no Medline e Lilacs. Resultados: Dentre os efeitos colaterais que surgem em curto prazo, prevalecem a redução de apetite, insônia, cefaléia e dor abdominal, sendo a maioria autolimitada, dose-dependente e de média intensidade. Dentre aqueles em longo prazo, são descritas alterações discretas de pressão arterial e freqüência cardíaca e uma possível discreta diminuição da estatura. O abuso e a dependência ao medicamento são observados muito raramente. Conclusões: O perfil de efeitos colaterais do metilfenidato é seguro, não parecendo justificar o seu uso constrito no Brasil, ante os benefícios robustos amplamente demonstrados na literatura.

Palavras-chave : Metilfenidato, TDAH, déficit de atenção com hiperatividade/ impulsividade.

\begin{abstract}
Objective: To review main short-term and long-term side effects of methylphenidate in the treatment of Attention-Deficit Disorder in children. Source of data: A 12-year review in Medline and Lilacs. Results: Among those side effects showed in the short-term, anorexia, insomnia, headache and abdominal pain are the most common ones, being the majority self-limited, dose-dependent and of medium intensity. Long-term side effects consist of mild blood pressure and heart rate increase and possible light growth suppression effect. Abuse and dependency are very rare. Conclusions: Methylphenidate side effects profile is safe and does not seem to justify its restricted use in Brazil, considering its solid benefits widely demonstrated in literature.
\end{abstract}

Keywords : Methylphenidate, ADHD, Attention-deficit Disorder with Hyperactivity.

Recebido: 07/01/2004 - Aceito: 09/03/2004

1 Mestrando em neurologia - Universidade Federal do Rio de Janeiro (UFRJ), pesquisador do Grupo de Estudos do Déficit de Atenção (GEDA) do IPUB/UFRJ

2 Professor-adjunto da UFRJ, Coordenador do GEDA - Grupo de Estudos do Déficit de Atenção do IPUB/UFRJ

Endereço para correspondência: Giuseppe Pastura, Instituto de Psiquiatria da UFRJ, Av. Venceslau Brás, 71, Fundos. 22290-140, Rio de Janeiro - RJ, Brasil, e-mail: giuseppepastura@ig.com.br 


\section{Introdução}

De acordo com a quarta edição do Diagnostic and Statistical Manual of Mental Disorders (American Psychiatric Association 1994), DSM-4, o Transtorno do déficit de atenção com hiperatividade/ impulsividade (TDAH) consiste em um padrão persistente de desatenção e/ou hiperatividade, mais freqüente e grave do que aquele comumente encontrado em crianças de nível de desenvolvimento semelhante. Surge antes dos sete anos de idade e interfere em pelo menos duas áreas de atuação da criança, como lar, colégio e grupo de amigos. Trata-se de enfermidade freqüentemente subdiagnosticada pelo pediatra. Ocorrendo a suspeita clínica, é importante o pronto encaminhamento para diagnóstico, uma vez que o tratamento farmacológico se impõe.

Um grande estudo randomizado iniciado em meados dos anos 1990 pelo Cooperative Group Multimodal Treatment Study of Children with ADHD (conhecido simplesmente como MTA) mostrou que a psicoterapia isolada, apesar de melhorar os sintomas de baixa auto-estima e sociabilidade, não foi eficaz no tratamento dos sintomas cardinais do transtorno. $\mathrm{O}$ uso de psicoestimulantes, como o metilfenidato, com ou sem psicoterapia associada, foi a melhor estratégia para o tratamento desses pacientes (Greenhill et al., 1996; The MTA Cooperative Group 1999). Existe uma considerável quantidade de dados atestando a segurança e eficácia do metilfenidato (Conners, 2002). Os efeitos mais robustos (effect size) demonstrados ocorrem na esfera do comportamento e da atenção, com índice terapêutico em torno de 0,8; os menores efeitos ocorrem no desempenho acadêmico, com índices terapêuticos em torno de 0,4 a 0,5 (Conners, 2002). A eficácia clínica do metilfenidato já foi comprovada em mais de 1.500 estudos clínicos nos últimos 40 anos (Conners, 2002).

\section{0 metilfenidato}

Drogas psicoestimulantes são utilizadas no tratamento de crianças e adolescentes desde a década de 1930 (Bradley, 1938). Seu mecanismo de ação é o estímulo de receptores alfa e beta-adrenérgicos diretamente, ou a liberação de dopamina e noradrenalina dos terminais sinápticos, indiretamente. Seu início de ação dá-se em 30 minutos, com pico em uma a duas horas, e meia-vida de duas a três horas (Bennett et al., 1999).
São três as hipóteses acerca do papel dos receptores alfa-adrenérgicos na fisiopatologia do transtorno (Newcorn et al., 1998):

1. Sendo responsável pelo processamento seletivo de informações recebidas, o locus ceruleus teria papel importante na atenção. Receptores alfa-adrenérgicos nele localizados são responsáveis pela modulação da resposta dessa estrutura aos distratores internos e externos. Uma disfunção nestes receptores faz com que o locus ceruleus reaja a qualquer estímulo e não mantenha atenção sustentada a um só estímulo;

2. Outra estrutura envolvida na atenção é o córtex parietal posterior através de fibras noradrenérgicas procedentes do locus ceruleus. O estímulo de tais fibras manteria o córtex parietal posterior pronto a responder a novos estímulos. O mau funcionamento dessa região cerebral explicaria o déficit de atenção;

3. Neurônios noradrenérgicos localizados no córtex pré-frontal estimulariam esta região a processar estímulos relevantes, inibir estímulos irrelevantes e restringir o comportamento hiperativo. Logo, sua disfunção traria dificuldades de atenção.

\section{Os efeitos colaterais em curto prazo}

Em estudo duplo-cego controlado acerca da freqüência de efeitos colaterais de metilfenidato em crianças com TDAH, Barkley e cols. (1990) descreveram 17 sintomas mais comuns em curto prazo. A tabela 1 apre-

Tabela 1: Freqüência de aparecimento de efeitos colaterais de metilfenidato em pacientes em uso de placebo, baixas $(0,3 \mathrm{mg} / \mathrm{kg})$ e altas $(0,5 \mathrm{mg} / \mathrm{kg})$ doses de metilfenidato.

\begin{tabular}{lccc}
\hline Efeito colateral & Placebo & Baixa dose & Alta dose \\
\hline Diminuição de apetite & 15 & 52 & 56 \\
\hline Insônia & 40 & 62 & 68 \\
\hline Dor abdominal & 18 & 39 & 35 \\
\hline Cefaléia & 11 & 26 & 21 \\
\hline Propensão ao choro & 49 & 59 & 54 \\
\hline Tiques & 18 & 18 & 28 \\
\hline Tonteira & 4 & 10 & 7 \\
\hline Náuseas & 18 & 23 & 20 \\
\hline Roer unhas & 22 & 26 & 29 \\
\hline Falar pouco & 16 & 20 & 22 \\
\hline Ansiedade & 58 & 58 & 52 \\
\hline Desinteresse & 18 & 18 & 15 \\
\hline Euforia & 41 & 34 & 43 \\
\hline Irritabilidade & 72 & 65 & 66 \\
\hline Pesadelo & 20 & 20 & 21 \\
\hline Tristeza & 43 & 48 & 41 \\
\hline “Olhar parado" & 40 & 38 & 38 \\
\hline
\end{tabular}

Modificado de Barkley RA, McMurray MB, Edelbrock CS et al. Side effects of Methylphenidate in Children with Attention Deficit Hyperactivity Disorder: a Systemic Placebo-controlled Evaluation. Pediatrics 1990; 86(2): 184-92. 
senta os efeitos colaterais em ordem de freqüência de aparecimento. Os mesmos são comparados nos pacientes em uso de placebo, baixas $(0,3 \mathrm{mg} / \mathrm{kg})$ e altas $(0,5 \mathrm{mg} / \mathrm{kg})$ doses de metilfenidato.

Observa-se que redução de apetite e insônia são os principais efeitos colaterais do metilfenidato. Destacam-se também dor abdominal e cefaléia. Sintomas comumente descritos como sendo causados pelo fármaco podem na verdade ser atribuídos à doença, tais como ansiedade, tristeza, desinteresse e "olhar parado”, uma vez que suas freqüências caíram com o uso de metilfenidato em relação ao grupo-controle. Efron et al. (1997), em estudo duplo-cego, também reforçam a observação feita por Ahmann e cols. (1993) de que certos sintomas tidos como efeitos colaterais da droga são, na verdade, características da população de portadores do distúrbio em questão.

Observou-se também que apenas metade da amostra estudada apresentou efeitos colaterais e, destes, nenhum passou do grau moderado de gravidade, o que vem ao encontro da opinião comum de que os efeitos colaterais dos psicoestimulantes são dose-dependentes e também desaparecem ou diminuem com o tempo ou com a redução da dose (Barkley et al., 1990; Adesman e Morgan, 1999). Outro estudo, duplo-cego controlado, obteve resultados distintos sobre a freqüência de efeitos colaterais de metilfenidato em crianças com TDAH. Ahmann et al. (1993) encontraram que, dentre as queixas mais comuns, somente redução de apetite é dose-dependente. Insônia, cefaléia, dor abdominal e tontura não foram influenciadas pela quantidade ingerida. Quanto à redução do apetite, é importante ressaltar com os pais a efemeridade do fenômeno e incentivar o uso de alimentos mais calóricos e polivitamínicos que compensem a perda de peso e a baixa ingesta alimentar (Adesman e Morgan, 1999; Bennett et al., 1999).

Embora alguns estudos sugiram benefícios do uso de psicoestimulantes em pacientes com TDAH e epilepsia, seu uso sempre foi cauteloso nesses pacientes devido à redução do limiar convulsivo causado por esses fármacos (Handen et al., 1990; Wilens e Biederman, 1992). Gross-Tsur e cols. (1996) demonstraram em estudo duplo-cego controlado que o metilfenidato é seguro para o uso em crianças epiléticas. Nesse trabalho, 30 pacientes com transtorno do déficit de atenção com hiperatividade/impulsividade e epilepsia foram analisados. Dos 25 que estavam sem crises ao iniciar o uso de metilfenidato, nenhum desenvolveu crises convulsivas. Dos cinco que não estavam assintomáticos no início do estudo, três aumentaram o número de crises e dois não tiveram alteração de seu status. Cerca de $70 \%$ dos pacientes obtiveram melhora de sintomatologia com o uso de metilfenidato.

Paralelamente à redução de apetite, tem-se a dor abdominal que pode igualmente comprometer a ingesta alimentar. Pode ser difícil contorná-la, porém deve-se orientar os pais a oferecer o metilfenidato junto com as refeições e reduzir a dosagem da droga temporariamente. Mais uma vez, é importante reafirmar a natureza temporária do efeito colateral aos invariavelmente aflitos pais que procuram o médico sem saber mais o que fazer para que seus filhos se alimentem adequadamente (Adesman e Morgan, 1999; Bennett et al., 1999).

A cefaléia, quando ocorre, deve ser contornada com o uso de analgésicos. É sempre importante um exame físico completo da criança a fim de descartar outras patologias pediátricas que possam estar causando cefaléia: hipertensão intracraniana, glomerulonefrite aguda com aumentos de pressão arterial etc. Quando o efeito é unicamente relacionado à droga, em poucos dias a semanas o desaparecimento é observado (Adesman e Morgan, 1999; Bennett et al., 1999).

Quanto à insônia, deve-se evitar o uso da medicação muito próxima ao horário em que o paciente vai deitar-se e também fornecer doses mais altas da medicação pela manhã e menores próximo ao final do dia (Adesman e Morgan, 1999; Bennett et al., 1999).

Em geral, tiques não são desencadeados pelo metilfenidato, sendo que pode até mesmo ocorrer melhora dos mesmos com o uso do fármaco. Porém, em caso de surgimento ou piora dos tiques em uso do metilfenidato, este deve ser suspenso. Na maioria dos casos, os tiques remitem ao tomar-se essa conduta (Adesman e Morgan, 1999; Bennett et al., 1999; Law e Schachar, 1999).

Psicose causada pelo metilfenidato é rara e exige interrupção imediata da terapêutica (Cherland e Fitzpatrick, 1999). Schteinschnaider et al. descreveram um caso de arterite cerebral relacionado ao fármaco cursando com grave alteração comportamental inicial (Schteinschnaider et al., 2000).

Rebote comportamental, definido como uma deterioração dos sintomas à medida que o efeito da medicação está terminando, foi descrito por Johnston et al. (1988). Seu manejo é feito espaçando-se as doses da medicação ao longo do dia, de modo que o nível sérico se mantenha o mais estável possível ao longo do tempo, evitando concentrações muito baixas. O metilfenidato de longa ação ou as formulações de liberação controlada e prolongada seriam, segundo Adesman e Morgan (1999), a escolha para estas situações. 
A seguir, são apresentadas algumas estratégias gerais para a administração dos efeitos colaterais do metilfenidato, segundo Adesman e Morgan (1999):

1. Observar se o efeito colateral investigado se deve realmente ao metilfenidato e não a qualquer outra condição médica. Por exemplo: dor abdominal causada por verminose;

2. Sempre que possível, reduzir ou suspender temporariamente a medicação, de modo a aguardar o fim do efeito indesejado e comprovar que este se deve realmente ao fármaco. Muitas vezes, a reintrodução do metilfenidato não se acompanha do efeito colateral ou este ressurge mais leve que anteriormente;

3. Oferecer a medicação com alimento;

4. Em caso de piora dos efeitos durante o pico da medicação, trocar para metilfenidato de liberação lenta;

5. Em caso de perda de apetite, fazer uso de metilfenidato de curta ação, o que facilita o manejo do tempo de ação da droga;

6. Tentar períodos de férias da medicação (feriados, finais de semana e férias escolares) de modo a evitar efeitos colaterais nesse período.

\section{Efeitos colaterais em longo prazo}

Em longo prazo, são três os efeitos colaterais de maior importância do metilfenidato: dependência, efeitos cardiovasculares e possível redução da estatura.

Dependência medicamentosa do uso do metilfenidato é um risco mais teórico do que prático. Geralmente, o paciente com TDAH consegue um bem-estar muito grande ao utilizar a medicação, o que, na verdade, é um estímulo para manter seu tratamento de forma adequada. A farmacocinética do medicamento, com início relativamente lento de ação e pico sérico em uma hora, torna menos provável o abuso para fins recreativos. $\mathrm{O}$ risco de abuso pelo paciente é considerado raro, porém há relato anedótico de uso indevido por parte de familiares e amigos (Llana e Crismon, 1999; Klein-Schwartz, 2002)

Estudo publicado por Klein-Schwartz (2002) descreve as principais manifestações clínicas causadas pela overdose de metilfenidato. São elas: agitação, crise convulsiva, alucinações, psicose, letargia, tonteira, taquicardia, hipertensão e hipertermia. Agitação, delírio e crise convulsiva podem ser tratados com benzodiazepínicos. Antipsicóticos são utilizados nos casos de alucinações. Bloqueadores do canal de cálcio e antagonistas alfa-adrenérgicos são recomendados para o controle da hipertensão. Lavagem gástrica é preconizada nos casos de ingesta oral maciça.
Os efeitos cardiovasculares do metilfenidato são pontuais e transitórios. Logo após o uso da medicação, pode-se observar pequena elevação da pressão arterial, freqüência cardíaca e respiratória, porém tais alterações não se sustentam ao longo do tempo (Zeiner, 1995; Bennett et al., 1999; Findling et al., 2001).

Quanto ao efeito de redução da estatura final de crianças com TDAH que fizeram uso de metilfenidato, encontramos dados conflituosos nos artigos disponíveis na literatura. Gittelman-Klein et al. (1998) em estudo controlado, observaram que pacientes que fizeram uso de metilfenidato por dois verões consecutivos sem férias do medicamento tiveram redução de $1,5 \mathrm{~cm}$ em comparação com os controles que tiveram férias da medicação. Já Kramer et al. (2000) demonstraram que pacientes que fizeram uso de metilfenidato não tinham sua estatura alterada se comparados ao alvo genético, à população em geral ou aos controles. Alguns indivíduos que apresentaram náuseas/vômitos e fizeram uso de altas doses de metilfenidato tiveram redução de estatura final. Spencer et al. (1996), em estudo controlado, observaram que o déficit estatural de crianças com TDAH deve-se ao próprio transtorno e não à medicação psicoestimulante utilizada. No estudo do MTA, documentou-se uma discreta redução da estatura e do peso ( 1 a $1,5 \mathrm{~cm} /$ ano e 1 a $2 \mathrm{~kg} /$ ano, respectivamente), que era dose-dependente. Esta redução pode não ser observada quando comparados a uma curva normal de crescimento, mas poderia afetar a estatura final caso não ocorra uma aceleração posterior ao final da adolescência.

\section{Comentários e conclusões}

O metilfenidato pode ser considerado medicação clinicamente segura no tratamento do TDAH, apresentando um perfil bastante satisfatório de efeitos colaterais. Aqueles ocorrendo em curto prazo são de pequena gravidade, autolimitados, dose-dependentes e facilmente contornáveis pelo médico. Embora menos estudados, os efeitos colaterais em longo prazo não são considerados como clinicamente graves, à exceção da dependência, fenômeno apenas muito raramente observado.

O uso bastante restrito do metilfenidato no nosso meio não encontra respaldo na literatura científi$\mathrm{ca}$, que deve nortear a medicina baseada em evidências. É razoável supor que tal uso esteja relacionado à desinformação acerca do medicamento. 


\section{Referências bibliográficas}

Adesman, A.R.; Morgan, A.M. - Management of Stimulant Medications in Children with Attention-Deficit/hyperactivity Disorder. Pediatr Clin North Am 46: 945-63, 1999.

Ahmann, P.A.; Waltonen, S.J.; Olson, K.A. et AL. - Placebo-controlled Evaluation of Ritalin Side Effects. Pediatrics 91(6): 1101-6, 1993.

American Psychiatric Association: - Diagnostic and Statistical Manual of Mental Disorders, ed.4. American Psychiatric Association, Washington, 1994.

Barkley, R.A.; McMurray, M.B.; Edelbrock, C.S. et AL. - Side Effects of Methylphenidate in Children with Attention Deficit Hyperactivity Disorder: a Systemic Placebo-controlled Evaluation. Pediatrics 86(2): 184-92, 1990.

Bennett, F.C.; Brown, R.T.; Craver, J.; Anderson, D. - Stimulant Medication for the Child with Attention-Deficit/hyperactivity Disorder. Pediatr Clin North Am 46:929-44, 1999.

Bradley, C. - The Behavior of Children receiving Benzedrine. Am J Psy chiatry 94: 577-85, 1938.

Cherland, E.; Fitzpatrick, R. - Psychotic Side effects of Psychostimulants: a 5-year Review. Can J Psychiatry 44(8): 811-3, 1999.

ConNERs, C.K. - Forty Years of Methylphenidate Treatment in AttentionDeficit Hyperactivity Disorder. J Att Dis 6 (suppl 1): S17-S30, 2002.

EFron, D.; JARMAn, F.; BARKER, M. - Side Effects of Methylphenidate and Dexamphetamine in Children with Attention-Deficit Hyperactivity Disorder: A double-blind, crossover Trial. Pediatrics 100 (4): 662 6, 1997.

Findling, R.L.; Short, E.J.; Manos, M.J. - Short-term Cardiovascular Effects of Methylphenidate and Adderall. J Am Acad Child Adolesc Psychiatry 40 (5): 525-9, 2001.

Gittelman, K.R.; Manuzza, S. - Hyperactive Boys almost Grown up III Methylphenidate Effects on Ultimate Height. Arch Gen Psychiatry 45: $1131,1998$.

Greenhill, L.L.; Abikoff, H.B.; Arnold, L.E. et AL. - Medication Treatment Strategies in the MTA Study: Relevance to Clinicians and Researchers. J am Acad Child Adolesc Psychiatry 35(10): 1304-13, 1996.

Gross, T.V.; Manor, O.; Van Der Meere, J. et AL. - Epilepsy and Attention-
Deficit Hyperactivity Disorder: Is Methylphenidate Safe and Effective? J Pediatrics 130 (4): 670-4, 1996.

Handen, B.L.; Breaux, A.M.; Gosuing, A. et al. - Efficacy of Methylphenidate among Mentally Retarded Children with Attention-Deficit Hyperactivity Disorder. Pediatrics 86: 922-9, 1990.

Jonhston, C.; Pelham, W.E.; HozA, J. et al. - Psychostimulant Rebound in Attention Deficit Disordered Boys. J Am Acad Child Adolesc Psychiatry 29: 719, 1988.

Klein-Schwartz, W. - Abuse and Toxicity of Methylphenidate. Curr Opin Pediatr 14(2): 219-23, 2002

Kramer, J.R.; Loney, J.; Ponto, L.B. et al. - Predictors of Adult Height and Weight in boys Treated with Methylphenidate for Childhood Behavior Problems. J Am Acad Child Adolesc Psychiatry 39(4): 517-24, 2000.

LAW, S.F.; Schachar, R.J. - Do Typical Clinical Doses of Methylphenidate cause Tics in Children Treated for Attention-Deficit Hyperactivity Disorder? J Am Acad Child Adolesc Psychiatry 38 (8): 944-51, 1999.

LLANA, M.E.; Crismon, M.L. - Methylphenidate: Increased Abuse or Appropriate Use? J Am Pharm Assoc 39(4): 526-30, 1999.

NewCorn, J.H.; Schulz, K.; Harrison, M. ET AL. - Alfa-2 Adrenergic Agonists Pediatr Clin North Am 45(5): 1099-22, 1998.

Schteinschnaider, A.; Plaghos, L.L.; Garbugino, S. et al. - Cerebral Arteritis following Methylphenidate Use. J Child Neurol 15(4): 265-7, 2000.

Spencer, T.J.; Biederman, J.; Harding, M. ET AL. - Growth Deficits in ADHD Children Revisited: Evidence for Disorder-Associated Growth Delays? J Am Acad Adolesc Psychiatry 35 (11): 1460-68, 1996.

The Mta Cooperative Group. Multimodal Treatement Study of Children with ADHD. A 14-month Randomized Clinical Trial of Treatment Strategies for Attention-Deficit/ hyperactivity Disorder. Arch Gen Psychyatry 56 (12): 11073-86, 1999.

WiLENS, T.E.; BIEDERMAN, J. - The Stimulants: Pediatric Psychopharmachology. Psychiatr Clin North Am 15: 191-222, 1992.

ZeIner, P. - Body Growth and Cardiovascular Function after Extended $(1,75)$ years with Methylphenidate in Boys with Attention Deficit Hyperactivity Disorder. Journal of Child and Adolescent Psychopharmacology 5:129, 1995. 